André Elias Mazawi

Université de la Colombie-Britannique

(Canada)

\section{Colonialismes, adversités et statut apatride: La condition enseignante dans le contexte palestinien}

\author{
Colonialism, adversities, and statelessness:
} Teachers and teaching in the Palestinian context

doi:10.18162/fp.2018.467

\section{ésumé}

L'essor de la condition enseignante palestinienne a eu lieu au long de plus d'un siècle parsemé de colonisation et de résistance. Le défi principal est de comprendre comment les constructions vernaculaires de la condition enseignante palestinienne se positionnent les unes par rapport aux autres, et par rapport à un territoire et à un champ de pouvoir de plus en plus fragmentés, attisant des luttes autour d'imaginaires collectifs contestés.

\section{Mots-clés}

Colonialisme, apatride, condition enseignante, fonction enseignante, Palestiniens, adversité.

\section{Abstract}

The evolution of the teaching force happened over more than a century of colonialism and resistance.

The main challenge is to understand how the vernacular constructions of what teaching stands for are positioned in relation to each other, over an increasingly fragmented territory and field of power, and how these constructions interface with political struggles around contested collective imaginaries.

\section{Keywords}

Colonialism, statelessness, teaching condition, teaching function, Palestinians, adversity.

\section{Introduction}

Mes réflexions sur le travail enseignant sont marquées par des expériences vécues, en tant que Palestinien arabe, dans le cadre d'institutions scolaires, universitaires et de formation initiale en Israël et dans la Cisjordanie occupée. Jusqu'à présent, les trames qui imprégnèrent le travail enseignant palestinien depuis la fin de la période ottomane en Palestine (1917), durant la colonisation britannique (1917-1948), et depuis la Nakba de 1948 - cette expérience de dépossession et d'exil des Palestiniens à la suite de la partition de la Palestine et de la création de l'État d'Israël (Masalha, 2012) - n'ont reçu qu'une attention marginale dans la recherche.

Le présent article cherche à comprendre les expériences vécues des enseignants et enseignantes dans la société palestinienne, leurs choix et pratiques dans l'école et à l'extérieur de celle-ci, sous différents contextes d'adversité. L'objectif est de problématiser les discours réductionnistes sur le professionnalisme enseignant qui mettent l'accent sur des pratiques certifiables, sans pour autant souligner l'importance des conditions de liberté et de justice qui doivent prévaloir pour donner un sens à ces pratiques. Je maintiens que la dimension « professionnelle » du travail enseignant ne peut se définir qu'en la plaçant sur l'arrière-fond des enjeux sociopolitiques qui permettent de réfracter son "noyau normatif qui donne sens à cette activité » (Tardif, 2014, p. 23).

\section{Profession entre « fonction » et « condition » enseignante}

Le statut professionnel de la fonction enseignante est contesté. Pour certains, la fonction enseignante représente un métier publiquement exercé, reposant sur une formation et une expérience acquises par rapport à un savoir-faire et dans un cadre institutionnel spécialisé. 
D'autres soutiennent que le corps enseignant a un pouvoir limité sur l'articulation des connaissances et pratiques professionnelles. Ce sont des organes régulateurs, situés en dehors du champ éducatif, qui colonisent les normes professionnelles par la certification de « compétences » (Oolbekkink-Marchand, Hadar, Smith, Helleve et Ulvik, 2017; Sachs, 2016; Wu, Cheung et Chan, 2017). Nonobstant, ces débats sont déconnectés des expériences vécues dans les sociétés du Sud, y compris dans la société palestinienne. Ils imposent «des discours mondialisés » sur les savoirs scolaires et les normes professionnelles, véhiculés par des organisations internationales contrôlées par le Nord (Tsehaye, 2015). Les politiques qui en découlent attisent souvent des conflits violents qui accentuent la précarité de la fonction enseignante en ce qui a trait au recrutement, à la rémunération et à la formation (Lanoue, 2006).

Pour y remédier, je m’inspire des travaux de Tardif (2013) sur l'évolution de l'enseignement au Québec, et de Fairclough (2007) sur l'enseignement dans les communautés noires aux États-Unis. Malgré les différences radicales entre ces contextes, ces études me permettent de distinguer entre la notion de «fonction enseignante" dans ses attributs formellement professionnalisants, et la notion de « condition enseignante» dans ses réalités vécues ancrées dans « l'esprit du temps » (Zeitgeist), c'està-dire dans le climat économique, politique, socioculturel et syndical dans lequel s'inscrit l'action éducative et les luttes qui l'attisent. Tardif (2014), qui contraste "rhétorique professionnalisante » et " réalités professionnelles », marque cette distinction, tout en nous rappelant que "l'enseignement est d'abord et avant tout un travail relationnel entre des êtres humains » (p. 23). Pour lui, « l'autonomie, le respect, la liberté pédagogique et le professionnalisme authentique » sont des acquis qui exigent à l'enseignant et à l'enseignante « de mobiliser un large spectre de savoirs, de ressources et d'habiletés qui couvre en fait les diverses modalités de l'interaction humaine : affective, normative, intellectuelle, culturelle, langagière, spirituelle» (p. 23). Ce cadrage place l'enseignement au cœur du bic et nunc de toute expérience de «l'agir» qui, comme le note Arendt (1995), représente une capacité insondable de l'être humain de "pouvoir-commencer ", de passer de l'être à l'acte, malgré les défis, pour régénérer des espaces relationnels alternatifs en vue de changer le monde. C'est cette capacité de mobilisation située - ce " pouvoir-commencer»-des enseignants et enseignantes que je vise à comprendre dans le contexte palestinien, dans ses espaces sociaux, politiques et géographiques en mutation constante depuis plus d'un siècle, et dans lesquels des imaginaires collectifs d'un passé, et ceux d'un à-venir, informent et forgent les pratiques. L'étude de Fairclough (2007) illustre la pertinence d'un tel cadrage. Fairclough observe que "la demande pour des enseignants noirs [aux États-Unis au début du $\mathrm{XX}^{\mathrm{e}}$ siècle] était soutenue par un désir de leadership noir et d'autonomie culturelle » (p. 67). Ainsi, « le lien entre l'éducation et la politique était brutalement clair. Les enseignants noirs étaient en ligne de front dans les batailles sur l'éducation » (p. 7). Ils ont favorisé « un sens plus général de fierté raciale qui joua un rôle important - si envahissant qu'il était souvent tenu pour acquis - dans la motivation du mouvement pour les droits civiques» (p. 388) à partir des années 1950. Fonction et condition enseignante sont donc imbriquées, «structurées » et «structurantes » (pour reprendre Bourdieu) par rapport au champ politique.

Sur cet arrière-fond conceptuel, deux questions interdépendantes guident ma réflexion sur la condition enseignante palestinienne. 
Premièrement, dans des contextes d'adversité coloniale, comme en est le cas palestinien, professionnalisme enseignant et aspirations collectives s'alimentent réciproquement. Cela nécessite une sensibilité conceptuelle aux enjeux multiformes entre l'action éducative dans la salle de classe et les luttes politiques dans lesquelles s'insèrent les enseignants et enseignantes en son dehors. L'étude de ces enjeux requiert un accent sur les interactions réciproques entre vie " publique » et " privée ", comme données complémentaires, pour comprendre les constructions qui sous-tendent le professionnalisme enseignant dans un contexte donné. Dans le contexte palestinien, comment se jouent les tensions entre fonction et condition enseignante en termes d'enjeux entre pratiques à l'intérieur de l'école et de la salle de classe, et d'insertion politique des enseignants et enseignantes en dehors de ces lieux?

Deuxièmement, dans les contextes d'adversité, la violence milite contre la capacité de l'agir de la condition enseignante. Les pertes de vie, les déplacements forcés, l'exil, la dépossession et la destruction des écoles sont monnaie courante dans de nombreux pays du Sud (Lanoue, 2006; Tawil, 1998), y compris dans le contexte palestinien (Veronese, Pepe, Dagdukee et Yaghi, 2018). Pourtant, cette violence est considérée sous l'angle de l'" aide humanitaire " alors que les lectures qu'en font les enseignants et enseignantes sont marginalisées. Dans le contexte palestinien, la violence (sous forme d'exil, et l'expérience de réfugié et de dépossession associée avec la Nakba et la colonisation israélienne) attise des composantes existentielles, identitaires et épistémiques. Ces composantes se manifestent, entre autres, dans les lectures « à contrepoint » (Said, 1994) qu'en font des enseignants et enseignantes, comme agents actifs, dans la construction d'imaginaires collectifs par rapport auxquels ces agents se positionnent. L'étude de ces lectures nous instruirait non seulement sur les vernaculaires professionnels qui se forgent au fil des circonstances, mais aussi sur les espaces relationnels alternatifs que les enseignants et enseignantes tentent d'articuler. Dans le contexte palestinien, quels espaces relationnels alternatifs les enseignants et enseignantes tentent-ils d'articuler dans leurs lectures des conditions d'adversité vécues?

\section{Démarche méthodologique}

Ma démarche méthodologique est exploratoire et interprétative. Elle s'appuie sur l'analyse de quatre catégories distinctes de matériaux :

a) des textes érudits sur le travail enseignant palestinien. Ces textes me permettent d'établir les éléments clés - d'ordre historique, sociologique et anthropologique - qui ont caractérisé le travail enseignant dans la société palestinienne arabe depuis la fin de la période ottomane au XIX ${ }^{\mathrm{e}}$ siècle et jusqu'à présent;

b) des données statistiques publiées officiellement par les agences statistiques israélienne et palestinienne qui permettent de retracer l'essor de la fonction enseignante et les clivages sociaux autour desquels elle a évolué au fil du temps;

c) des textes autobiographiques offrant une narration à la première personne des expériences vécues par des Palestiniens et des Palestiniennes qui ont commencé leur cheminement dans l'enseignement, ou qui ont eu une carrière enseignante. Ces textes situent l'expérience de l'enseignement sur l'arrière-fond des dynamiques sociales et politiques vécues par les protagonistes et les réseaux sociaux dans lesquels ils ou elles opéraient, dans la salle de classe et l'école, mais aussi hors de ces lieux. Je reconnais les limitations méthodologiques inhérentes à une analyse de 
textes autobiographiques composés ex post facto. Pour cette raison, je soumets ces textes à une analyse critique du discours par rapport à leurs contextes de production;

d) des documents d'archives qui comprennent une gamme de ressources comme des photographies, des œuvres d'art et des témoignages ou documents non publiés. Cette dernière catégorie n'a pas été pleinement incluse dans le présent article pour des raisons d'espace, quoique j’intègre certains aspects dans mon analyse, à titre illustratif.

Ces matériaux permettent d'établir les complémentarités et les ruptures qui se jouent entre pratiques enseignantes et champ politique. Dans l'intégration de tous ces matériaux, je suis inspiré par les travaux de Mills (1967), de Bateson (1989) et de Said (1994), qui consistent à « composer la vie » des enseignants et enseignantes, en reconstituant les imbrications, les ruptures et les continuités entre les pratiques et les choix faits à différentes jonctions de leurs vies. Une telle approche permet de réfracter des lectures critiques qui révèlent comment ces acteurs ou actrices « extraient » les significations qu'ils ou qu'elles attribuent à leurs expériences vécues - et les « lectures à contrepoint " qu'ils ou qu'elles en font - dans des contextes d'adversité, de vies chaotiques, non linéaires, dans des situations improvisées et désordonnées, dans lesquelles les aspects "privés " et "publics " sont entrelacés de manières multiformes.

\section{Colonisation et nationalisme}

La période de 1917 à 1948 donne lieu à l'expansion de la scolarisation gratuite, mais pas obligatoire, et à l'émergence d'une classe enseignante arabe, cela dans un contexte de colonisation britannique et sioniste de la Palestine. Je m'attarde ici aux tensions qui sous-tendent cette dynamique et à leurs effets sur la condition enseignante palestinienne.

Avant 1917, l'Empire ottoman avait introduit des écoles publiques où l'enseignement était offert en turc. En 1914-1915, les 98 écoles du gouvernement ottoman en Palestine employaient 234 enseignants et enseignantes pour 8248 élèves (dont $17 \%$ de filles), soit $11,5 \%$ des enfants arabes en âge de scolarisation (7 à 11 ans) (Tibawi, 1956, p. 270). Des écoles gérées par des communautés palestiniennes musulmanes (organisées autour de la madrasa et du kuttab dans les grandes mosquées) enseignaient en arabe.

D'autres écoles étaient gérées par des communautés palestiniennes chrétiennes et juives alors que des institutions missionnaires et religieuses chrétiennes, installées en Palestine, enseignaient dans différentes langues, dont le français et le russe. Cette diversité a amené Hauser, Lindner et Möller (2016) à observer qu'à travers le Levant l'éducation durant la période ottomane était " particulièrement dense par la concentration de différents établissements » (p. 14) et que « les enseignants se présentaient en modèles éducatifs vivants, tandis qu'ils étaient soumis à la censure organisationnelle » (p. 17). À partir de la fin du XIX siècle, l'éducation "moderne " "n'était pas imposée de l'extérieur, mais émergeait plutôt de ce réseau de rencontres enchevêtrées » (p. 21), en même temps que la politique éducative ottomane « usait de l'éducation comme outil pour standardiser et centraliser ses relations avec ses sujets ottomans» (p. 14). 
L'introduction d'écoles palestiniennes arabes locales, dotées d'un programme qui englobeles matières audelà des études religieuses, revient à des initiatives privées. En 1906,le Cheikh Muhammad Suleiman alSalih (décédé en 1940), de Jérusalem, fonda la Rawdat al-ma’arif al-wataniya ou «Jardin national [ou patriotique] des connaissances ", " la première école islamique qui développa un curriculum moderne » (Boullata,2009,p. 73).En 1909, Khalil al-Sakakini (né en 1878 et décédé en 1953) fonda Al-madrasa aldoustouriyya ou "École constitutionnelle » à Jérusalem, ouverte à toute communauté religieuse, dans l'esprit de l'éducation progressive et des réformes constitutionnelles ottomanes introduites en 1908 et inspirée des valeurs des Lumières et du progrès.

Sous la colonisation britannique, la langue arabe devint langue d'instruction. Les autorités coloniales fondèrent le Collège arabe de Jérusalem (jusqu'en 1927, Men's Teachers Training College) pour la formation initiale d'enseignants en langue arabe. Al-Sakakini en fut nommé directeur en 1919 (Davis, 2003). S'il est vrai que le Collège était une fondation élitiste, suivie bientôt par des collèges pour femmes, il n'est pas moins vrai qu'il devint un foyer d'activisme nationaliste. Soumis à des critiques "de la part d'éducateurs arabes et des leaders nationalistes » (p. 192), le Collège fut paralysé par des démonstrations antibritanniques en 1925, menant au départ de son deuxième directeur, Khalil Totah (né à Ramallah en 1886 et décédé aux États-Unis en 1955). S'opposant à la clôture du Collège durant les grèves, et face à l'activisme de ses étudiants et de certains professeurs, Totah écrivit :

Mon attitude vis-à-vis des grèrves arabes, qui sont trop fréquentes et généralement futiles, était négative. Je prêchais pour un travail ardu de la part des Arabes et non des grèves, s'ils voulaient tenir tête aux Sionistes. Je leur ai dit que la perte de leur travail chaque jour permettait aux Juifs de les devancer pour autant. Je ne voudrais pas rejoindre la foule bruyante dans les rues ou défiler avec la démonstration. (Ricks, 2008, p. 62)

Le départ de Totah souligna la difficulté de réconcilier les aspirations nationales arabes et les attentes qu'avait le gouvernement colonial de ses employés indigènes. Nonobstant, l'accès à l'école de la population arabe en âge de scolarisation passa de 11,5\% (en 1914, veille de l'occupation britannique) à 31,2 \% (en 1948, veille de la création d'Israël), restant bien inférieur à celui de la société juive (Tibawi, 1956, p. 270). Le nombre d'enseignants et d'enseignantes dans les écoles arabes publiques passa de 1036 en 1914-1915, à plus de 4600 en 1945-1948, consolidant une fonction enseignante, salariée par le gouvernement, professant en langue arabe. Son poids passa de 22,6 \% à 58,7 \% du corps enseignant dans les écoles publiques et non publiques réunies. Ceci dit, vers 1948, les enseignants et enseignantes dans la société palestinienne arabe ne représentaient que $39 \%$ de la force enseignante de Palestine, alors que les Arabes représentaient $70 \%$ de la population. Lintensification du conflit politique entre Palestiniens arabes et Sionistes poussa un bon nombre d'entre eux à joindre des groupements militants. Abdul Latif Tibawi (né à Taybeh en 1910 - décédé à Londres en 1981) est l'un des rares cadres supérieurs palestiniens au ministère de l'Éducation; il a protesté contre la politique coloniale britannique en Palestine. Il nota que durant l'insurrection arabe de 1936-1939 « il n'y avait aucun pouvoir capable de contrôler toutes leurs activités tout le temps dans cette sphère de leur travail. Tout en se conformant à la lettre du syllabus officiellement publié, ils ne perdirent aucune occasion pour lui donner un esprit national qui est leur ». Arrêtés pour sédition, «beaucoup d'autres ont été maintenus dans des camps de détention ", alors que d'autres furent renvoyés (Tibawi, 1956, p. 197-199). 
La fonction enseignante dans les écoles arabes publiques dépendait entièrement $\mathrm{du}$ ministère de l'Éducation alors que les écoles sionistes et chrétiennes relevaient de leurs organisations autonomes respectives, l'Agence juive et les églises ou congrégations. Toute tentative d'altérer cette gouvernance inégale poussa les leaders sionistes et ceux des écoles chrétiennes à s'y opposer. Du côté sioniste, le secrétaire du comité culturel de l'Union des enseignants, Yaacov Klibansky (né en Lituanie en 1888 - décédé en Israël en 1950), publia sous son nom de plume, "Y. Ushpiz », un article dans lequel il critiqua les tentatives d'ingérence du gouvernement colonial dans les écoles sionistes, à l'instar de ce qui a été déjà fait « sans aucune opposition à l'éducation des Arabes »(Ushpiz, 1940, p. 207). Pour lui, l'introduction d'une nouvelle ordonnance sur la gouvernance scolaire ferait « écrouler le bâtiment tout entier que nous sommes en train de construire sur notre terre pour faire valoir notre renaissance sur la terre des aïeux ». Alors, « notre forteresse la plus précieuse - l'école - serait prise d'assaut étape par étape » (p. 208).

Deux projets nationaux - arabe et sioniste - bouillaient sous la colonisation britannique, atteignant leur paroxysme en 1947 avec la partition de la Palestine par l'Organisation des Nations Unies (ONU). Dans les dénouements qui attisèrent la $N a k b a$, les enseignants et enseignantes arabes furent pris dans un étau. D'une part, la domination des élites palestiniennes reléguait au second plan les apports de ces jeunes literati politisés, d'origines modestes, atténuant leur position sur le champ politique. D'autre part, la mainmise du colonialisme britannique sur l'école publique arabe les empêcha de se tailler un espace professionnel autonome. Leurs lectures à contrepoint, publiées dans la presse et sous forme de pamphlets, propagèrent pourtant un discours identitaire militant.

\section{Profession d'exilés, profession exilée}

La Nakba palestinienne signala l'entrée en scène des réfugiés palestiniens, comme acteurs politiques, particulièrement dans l'enseignement (Moussa, 1972). En 1948, on comptait environ 700000 réfugiés, appartenant pour la plupart aux classes sociales et rurales défavorisées, sur une population arabe de 1,3 million de personnes. La création de l'Office de secours et de travaux des Nations Unies pour les réfugiés de Palestine dans le Proche-Orient (UNRWA), en 1949, stabilisa leur statut dans la géopolitique régionale.

Les enseignants et enseignantes d'origine réfugiée étaient actifs dans le recrutement de membres soit pour l'Organisation de libération de la Palestine (OLP), fondée en 1964, soit pour des organismes qui l'ont précédée (Sayigh,1997). Un des cofondateurs du Fath, composante majeure de l'OLP, Salah Khalaf (né à Jaffa en 1933 - assassiné en Tunisie en 1991), raconta ses débuts à Gaza (sous contrôle égyptien), en 1957-1958, à l'âge de 24 ans, comme enseignant du primaire :

Licencié en philosophie et en psychologie [...] [en Égypte, je] pouvais donc aisément postuler pour un poste dans l'enseignement.

Les services de sécurité comprirent que ce nétait pas sans arrière-pensées que je cherchais à memployer à Gaza. [...] À ma surprise, on me nomma instituteur dans une école de filles, mesure exceptionnelle que l'on prenait généralement en guise de sanction. [...] Je compris que l'on tentait de m'isoler pour mempêcher d'avoir une activité politique quelconque... 
[...] Je ne me décourageais pas pour autant. Je décidai d'abuser de la position que joccupais pour me livrer ouvertement à l'agitation politique. [...] [J]'invitai les élèves de mes classes à constituer des groupes de formation civique que je nommai "comités patriotiques». Ils étaient chargés de susciter et d'animer des débats pendant les heures de cours. Six mois plus tard, en pleine année scolaire, la directrice de l'établissement m'informa que jeetais muté à une école primaire de garçons...

[...] Située en plein désert, hors de Gaza, [l'école de garçons] était fréquentée par les enfants de réfugiés palestiniens habitant les camps tout proches. Les classes étaient exiguës, surpeuplées (soixante à soixante-dix élèves par cours) et délabrées. En hiver, nous grelottions de froid tandis que la pluie s'infiltrait à travers de fragiles plafonds. En revanche tout était propice aux activités politiques auxquelles je comptais me livrer. [...] Ayant constitué un comité d'aide à la révolution algérienne [parmi les élèves], je leur demandai de contribuer, chacun selon ses moyens, à une collecte de fonds. (Khalaf, 1978, p. 51-56)

La lecture de Khalaf dépeint l'enseignant comme activiste engagé dans la promotion d'une conscience de classe sociale solidaire avec les peuples colonisés. Il faut placer cette lecture dans le contexte de la résistance palestinienne à l'occupation israélienne de la bande de Gaza et du Sinaï en 1956. Aussi importante fut la décolonisation africaine, dont l'Algérie représentait alors le cas emblématique. Pour Khalaf, la légitimité professionnelle de la fonction enseignante émanait d'une déontologie politique qui considère l'enseignant comme activiste politique et engagé. ${ }^{2}$

L'expérience de la condition enseignante était différente pour les Palestiniens employés dans les écoles des États du Golfe. Depuis les années 1950, l'exploitation des gisements pétroliers et de gaz offrait aux réfugiés palestiniens, aux Palestiniens de la Cisjordanie et de la bande de Gaza, et à des ressortissants arabes, des débouchés économiques considérables. Leila Khaled (née à Haïfa en 1944) raconte ainsi son travail au Koweït, où elle débuta comme enseignante, à l'âge de 19 ans, en 1963 :

Le seul aspect vivant de l'école était la multitude de dialectes «étrangers " que l'on y parlait. Les groupes les plus importants étaient constitués par les Égyptiens, les Palestiniens et les Syriens. [...] Souad, la directrice, [...] était la femme autoritaire par excellence et une parfaite petite bureaucrate à caractère obsessionnel [...] qui se prosternait devant le ministère de l'Éducation nationale tout en s'organisant pour discipliner ses collègues enseignants par l'intermédiaire d'un réseau d'informateurs.

[...] La directrice avait menacé de me faire renvoyer si je persistai à me produire vêtue de corsages à manches courtes. [...] Je persistai néanmoins et la menaçai même d'exposer encore plus largement mon corps aux regards.

[...] [Les] enfants présentaient tous les caractéristiques de la pauvreté culturelle d'une population bédouine et isolée, mais ils étaient intelligents et assimilaient les connaissances avec une grande facilité. Pour eux, notre école était la seule porte ouverte sur le monde extérieur. Il aurait fallu enseigner les rudiments de la vie aux enfants de cette communauté à croissance rapide, et les mettre en garde à l'esclavage urbain. Au lieu de cela, je leur enseignais l'anglais et les sciences. Pourtant, je ne laissais jamais passer une occasion de mettre en avant mes idées politiques. 
[...] [J]'injectais mon virus politique à petites doses. Je reliais tous les problèmes au monde qui nous entourait et, chaque fois que possible, à la question palestinienne.

[...] [Mutée en 1964 à une autre école], je constatai que l'on m'a fait baisser de grade: des classes intermédiaires, j'étais passée à l'enseignement élémentaire. Pendant cinq ans, je fus réduite au cours préparatoire et, ainsi, privée de mon audience d'adolescents et de la possibilité d'utiliser ma classe comme plate-forme politique. [...] Mes élèves étaient presque tous d'origine iranienne. Leurs parents avaient immigré au Koweit en quête de travail.

[...] Au niveau du corps enseignant, peu ou prou de tensions et pas d'accrochages avec d'autres collègues ou la direction. À partir de 1968, le directeur et un grand nombre de professeurs virèrent en faveur du Fath. Ceux d'entre nous qui soutenaient le Front populaire n'avaient pas de difficulté pour coexister avec eux. [...] [Je] ne révélai jamais publiquement mes véritables affinités politiques. J'avais, en outre, une petite expérience de l'enseignement, ce qui mévita les affrontements avec des fonctionnaires imbus de leurs fonctions. (Khaled, 1973, p. 89-92 et p. 95-97)

Durant son travail au Koweï, Khaled dit avoir connu « une période d'incubation intellectuelle et de réflexion ». Quelques-uns de ses collègues «menaient une vie hédonique ", d'autres " envoyaient une grande partie de leur traitement à leurs parents et menaient une vie plus austère » (p. 97). Son engagement au sein du Front populaire de libération de la Palestine (FPLP), à tendance marxisteléniniste, la confirma dans sa conviction que, « je ne suis plus une réfugiée car je suis une révolutionnaire » (p. 125). Au sein du FPLP, elle participera à l'enlèvement d'avions (1969-1970). Emprisonnée, elle fut libérée par le gouvernement britannique en échange d'otages.

L'expérience de Khaled rejoint celle de Khalaf par son activisme clandestin et l'engagement politique en classe. Khaled enseigna cependant dans des écoles qui servaient les enfants d'immigrants et ceux provenant de communautés en processus d'urbanisation. Sa lecture nous offre une perspective sur le vécu de jeunes femmes enseignantes en exil, où les Palestiniens représentaient $50 \%$ de l'ensemble de la fonction enseignante en 1965 au Koweït. Khaled visait à secouer les normes patriarcales, tout en mettant l'accent sur la lutte armée. Dans les écoles publiques du Golfe se brassaient donc des activistes palestiniens affiliés à différents mouvements idéologiques.

En contraste, la trajectoire de Jabra Ibrahim Jabra - poète, écrivain, et artiste - fut différente. De parents illettrés, Jabra (né à Bethléem en 1919 - décédé en Irak en 1994) fut éduqué à Bethléem et à Jérusalem. Diplômé du Collège arabe de Jérusalem en 1937, il obtint une bourse pour poursuivre ses études en littérature anglaise en Angleterre, avant de rejoindre le corps enseignant de l'école secondaire Rashidiya à Jérusalem en 1943. Prenant la route de l'exil sous le feu de la Nakba, il fut nommé à 29 ans à un poste d'enseignant dans un collège supérieur de Bagdad (Irak), dont la moitié de ses collègues était des réfugiés palestiniens :

On m’avait attribué un logement dans l'enceinte du collège où j'enseignais. Et puis j'aimais les étudiants, tous brillants esprits, triés sur le volet, en passe d'être expédiés dans des universités étrangères.

[...] Je lançai une société de débats au collège, une autre de musique et, lors des fêtes, je dansais la dabkeh avec les étudiants. Un an plus tard, je montais un groupe de théâtre et inaugurais un atelier pour les peintres amateurs. Toutes les conditions étaient ainsi réunies 
pour aborder 1'ère nouvelle qui s'ouvrait à nous, me disais-je. Je prêchais le changement, sans honte aucune. Nous avions été trompés et trahis par mille ans de déclin, victimes de notre belle et absurde rhétorique. Nous avions perdu la Palestine pour avoir tenté de combattre une force impitoyable avec pour seule arme une tradition d'un autre temps. II fallait tout changer. Et ce, de fond en comble, en commençant par changer de perspective. Adopter un regard nouveau sur la réalité. Une manière nouvelle de s'exprimer. Une approche, une représentation nouvelle de l'homme et du monde.

$[\ldots]$

Les professeurs palestiniens figuraient également en grand nombre dans les autres établissements supérieurs de la ville. Nouveaux venus tout comme moi, certains étaient des mathématiciens de premier rang sortis de Cambridge, ou encore des ingénieurs, des économistes ou des historiens. Chacun dans notre domaine, nous nous étions tacitement fixés pour objectif de maintenir un niveau d'enseignement de qualité. Nous changions souvent de programme afin d'offrir à nos étudiants des cours toujours plus stimulants. Nous entretenions avec ces derniers des relations informelles, fondées sur la confiance et l'échange, ce qui nous valait parfois les remontrances de l'administration. Cela n'a pas duré. En l'espace de trois ou quatre ans, nous nous étions de nouveau dispersés. (Jabra, 1997, p. 7)

La lecture à contrepoint qu'offre Jabra vise plutôt l'intérieur de la société palestinienne et arabe en vue de déclencher une renaissance d'une envergure socioculturelle.

Les lectures à contrepoint offertes par Khalaf, Khaled et Jabra - présentées ici à titre illustratif soulignent le caractère situé de la déontologie de la profession enseignante. L'activité enseignante émerge comme espace de conscientisation solidaire (Khalaf), mouvement révolutionnaire (Khaled), renaissance socioculturelle (Jabra) ou moyen de survie économique.

\section{L'exil intérieur}

Quelque 160000 Palestiniens restèrent à l'intérieur de ce qui devint l'État d'Israël (sur une population de 1,2 million d'habitants en 1948). Soumis jusqu'en 1966 à un gouvernement militaire, leur accès à la fonction enseignante dépendait d'un système de patronage politique et de surveillance sécuritaire auxquels furent soumises les écoles publiques «pour Arabes ». Sa'di (2014) montre que «le dépistage et la surveillance des candidats pour l'enseignement commença avec leur inscription au collège » de formation initiale. Des renseignements obtenus sur chaque candidat, provenant de fonctionnaires et collaborateurs, permettaient d'identifier les "éléments négatifs » avant leur admission (p. 123). En outre, des « informateurs étaient recrutés parmi les enseignants, les élèves (même à un jeune âge), les directeurs, les inspecteurs et les parents»(p.127). Cette surveillance rendit les enseignants et enseignantes « à la fois des sujets et des objets actifs de surveillance » (p. 130). Séparés au sein du syndicat professionnel, tout essai d'établir des listes professionnelles qui représenteraient les enseignants et enseignantes palestiniens était poursuivi de mesures disciplinaires (p. 132-134).

Entre 1948-1949 et 2014-2015, la main-d'œuvre enseignante palestinienne à l'intérieur d'Israël passa de 170 équivalents temps plein pour une population de 10005 étudiants à 31124 équivalents temps plein pour une population de 434759 étudiants, soit le quart des postes enseignants dans le pays (State 
of Israel, Central Bureau of Statistics, 2015). Le taux de féminisation passa de 3,8 \% à 70,6 \% au primaire et à 60,9\% au secondaire. Étant donné la discrimination institutionnalisée des Palestiniens, la fonction enseignante offre une filière économique et de mobilité à l'intérieur des communautés palestiniennes, surtout pour les femmes. En outre, cette condition enseignante est prise entre une conformité aux règlements et lois et un combat civique, dans le cadre d'organisations non gouvernementales, pour une éducation adaptée à la spécificité culturelle de la population palestinienne. Des législations israéliennes pénalisant la commémoration de la Nakba dans l'école amplifient l'exil épistémique et pédagogique du corps professoral (Mazawi, 2017), poussant les enseignants et enseignantes à ne pas discuter du conflit politique en classe de "peur d'ouvrir la bouche, personne ne veut avoir affaire avec l'établissement [1'État]»(Khoury-Watad, 2008, p. 273). Cette impuissance politique a été exprimée ainsi par un enseignant d'une école publique " pour Arabes » dans une communauté bédouine :

Quelqu'un [d'autre] détermine ce que nous devons enseigner. Cela a à faire avec la politique. Prenons l'histoire de notre peuple. Je ne peux pas l'enseigner à l'école. [...] Le Ministère me la détermine. Nos aspirations, nous devrions [pouvoir] construire notre programme scolaire, en arabe, religion, histoire. [...] Le programme scolaire actuel est très faible. Si nous voyageons dans un pays [arabe] voisin, nous réaliserons que nous ne connaissons pas la langue arabe. En ce qui concerne l'bistoire, je sais que les minorités à travers le monde construisent leurs propres programmes scolaires. Pas nous, nous ne le pouvons pas. (Mazawi, 2005, p. 100)

De fait, cet enseignant déplorait l'absence d'un « pouvoir-commencer » dans la signification que lui prête Arendt (1995). Cette absence lui barre la possibilité, pour emprunter les mots de Tardif (2013), "de façonner une bonne part de l'avenir d'une société » (p. 5). Veronese et al. (2018) conclurent que les enseignants et enseignantes arabes en Israël « affirment que le système éducatif entrave leur travail et a un impact négatif sur leur vie» (p. 24).

\section{Enseigner sous occupation militaire}

L'occupation de la Cisjordanie et de la bande de Gaza par l'État d'Israël en juin 1967 déclencha une seconde vague d'exils parmi la population palestinienne. Un cadre de militaires israéliens assuma le contrôle du secteur éducatif, particulièrement la gestion directe des écoles palestiniennes «publiques ».

Entre 1967 et 1987, la fonction enseignante dans les écoles «publiques » passa d'environ 5000 à 10700 membres. La féminisation resta plus faible dans la bande de Gaza (autour d'un tiers) par rapport à la Cisjordanie (41,6 \%). Elle était plus élevée dans les écoles UNRWA et atteignit les deux tiers dans les écoles privées par rapport aux écoles publiques (Al-Zaroo, 1988; Royaume hachémite de Jordanie, Ministère de l'Éducation, 1966). La précarité matérielle de la condition enseignante était considérable. Les salaires restèrent attachés, non pas au coût de vie sous l'occupation israélienne, mais aux échelles de traitement en vigueur en Jordanie et en Égypte à la veille de l'occupation. Le personnel enseignant était dépourvu de toute «formation significative en cours d'emploi pour améliorer ses compétences et porter à jour les stratégies et méthodes d'enseignement »(UNESCO, 1995, p. 24). Selon l'étude de Graham-Brown (1984), les autorités militaires israéliennes empêchèrent la création de syndicats professionnels pour éviter «l'unification de tout groupe palestinien doté de conscience 
politique ». Toute grève «conduisit aux transferts punitifs des enseignants" (p. 79). En outre, les autorités militaires avaient aussi "interrogé des enseignants pour obtenir des informations sur des élèves actifs politiquement. À l'occasion, des enseignants furent emprisonnés pour avoir refusé de divulguer cette information» (p. 79). La profession enseignante manquait d'instruments collectifs pour déterminer ses normes éthiques et professionnelles.

La résistance contre l'occupation israélienne entraîna la participation de membres de la fonction enseignante. Du 30 octobre 1968 au 7 avril 1988, 30 enseignants furent exilés, dont cinq enseignantes. De juillet 1970 à juillet 1987 - à la veille de l'Intifada - 17 enseignants, dont cinq enseignantes, furent placés en résidence surveillée (Al-Zaroo, 1988). Avec l'irruption de la première Intifada (décembre 1987), des confrontations mortelles prirent lieu. Les autorités militaires imposèrent la clôture des établissements éducatifs par le biais dordres militaires. Des enseignants et enseignantes considérés comme "subversifs » furent licenciés, d'autres furent exilés, emprisonnés, pénalisés par une réduction salariale, ou bien mutés à la retraite. Durant la première année de l'Intifada, depuis sa veille le 27 octobre 1987 et jusqu'au 6 juillet 1988, 77 enseignants furent arrêtés, dont trois enseignantes (AlHaq, 1990; Al-Zaroo, 1988). Des enseignants et enseignantes mirent sur pied des filières clandestines d'études, coordonnées par des « comités populaires ", pour maintenir la scolarisation comme résistance non violente dans des maisons et des espaces privés. ${ }^{3}$

Sous l'Autorité palestinienne (AP), entre 1994 et 2016, la fonction enseignante dans les territoires occupés a plus que doublé ses effectifs, qui sont passés de 21054 à 55 160. Dans les écoles publiques, le nombre a presque triplé, représentant le plus grand secteur de la fonction publique de l'AP. Le taux de féminisation de l'ensemble de la population enseignante, qui était déjà marqué en 1994 (47,2 \%), dépassa en 2016 la marque des 57 \% dans les écoles publiques, des $65 \%$ dans les écoles UNRWA et des $75 \%$ dans les écoles privées (Palestinian Central Bureau of Statistics, and Ministry of Education and Higher Education, 1995; State of Palestine, Central Bureau of Statistics, 2016). Dans la même veine, le développement professionnel de la fonction enseignante prit un élan à partir des années 2000, dans le cadre d'un ministère de l'Éducation établi avec le soutien d'organisations internationales comme l'UNESCO. Cependant, si les capacités cruciales des enseignants et enseignantes de résister à l'occupation israélienne assurèrent la continuité de l'enseignement durant les longues périodes de clôture militaire sous l'Intifada, l'AP et son ministère " n'ont pas tenu compte de l'éducation clandestine en dehors des écoles qui s'est développée " pour construire sur cette expérience et régénérer la fonction enseignante (Tawil, 1998, p. 13). Plutôt, dans un contexte où plus de $60 \%$ de toute l'aide à l'éducation de base parvient des États-Unis, de l'Union européenne, de l'ONU, de la Banque mondiale et de l'Allemagne, la capacité des enseignants et enseignantes à exercer une influence sur les politiques éducatives adoptées reste limitée (Shinn, 2012, p. 614). À partir de 2005, une série de réformes ont été donc lancées pour la formation des enseignants palestiniens, en commençant par l'établissement d'un Institut national pour l'éducation et la formation. En 2008-2009, une initiative intitulée « Des systèmes de qualité pour des enseignants de qualité » fut introduite dans le cadre d'un programme financé par l'UNESCO, pour améliorer la qualité de la formation initiale et la formation continue. D'autres initiatives furent introduites, par exemple par la Banque mondiale en 2010, pour promouvoir la responsabilisation des enseignants et enseignantes à base de compétences « surveillées et gérées » par le ministère de l'Éducation. Ces initiatives se trouvèrent prises dans des engrenages bureaucratiques de l'AP, à la suite de l'accent mal placé sur le rôle des universités dans la formation continue, exacerbant 
la « confusion sur l'autorité et la responsabilité » en ce qui concerne « le développement de nouvelles connaissances et compétences pour les enseignants »(p. 626). À partir de 2007, la scission entre les mouvements du Hamas et du Fatah résulta en deux gouvernements palestiniens, à Gaza et à Ramallah respectivement, compliquant l'application de politiques éducatives cohérentes à travers les deux régions.

Sous l'effet d'une AP non souveraine et d'une occupation israélienne persistante, d'une part, les questions salariales sous-tendent les relations entre l'AP et les syndicats enseignants. Un accord sur les salaires conclu en 2013 n'empêcha pas l'irruption en 2016 d'une grève massive du corps enseignant en Cisjordanie. D'autre part, les enseignantes et enseignants sont pris dans les engrenages violents de l'occupation militaire israélienne qui dure depuis plus d'un demi-siècle. Leur maintien d'une routine scolaire est compromis par les incursions militaires dans les écoles et par les points de contrôle qui empêchent le mouvement libre des élèves et du corps enseignant (Shalhoub-Kevorkian, 2010). Sultana (2006) rapporte que, dans la ville de Hébron (Cisjordanie) - divisée entre colons israéliens et habitants palestiniens -, des membres de la fonction enseignante ont été battus et exposés aux tirs. D'autres étaient « incapables de se concentrer, oubliant souvent ce qu'ils voulaient enseigner car ils utilisaient beaucoup de leurs énergies et de leurs ressources personnelles pour éviter les soldats dans les rues, en faisant un détour pour arriver à école » (p. 58). Plus récemment, le ciblage d'institutions éducatives dans la bande de Gaza a été signalé durant le conflit du 7 juillet au 26 août 2014, qui opposa l'État d'Israël et des groupes armés dans la bande de Gaza : « 22 écoles ont été complètement détruites et 118 écoles endommagées ", avec 75 écoles de l'UNRWA endommagées, alors que ce territoire manquait déjà, avant le conflit, de " presque 200 écoles, avec des classes qui fonctionnaient à double-vacation ". Parmi les 2131 Palestiniens morts, 501 étaient des enfants. Le rapport estimait qu'avec la reprise des classes, en septembre 2014, « des centaines de milliers d'enfants ont besoin de support psychologique » (UNOCHA, 2014, p. 1) et que « les enseignants et le personnel éducatif (dont beaucoup souffraient de traumatisme aigu) seront étirés pour fournir le support requis pour faciliter le retour des enfants à l'école et pour fournir un support continu durant l'année scolaire »(UNOCHA, 2014, p. 3-4). Aliénés, les enseignants et enseignantes sont pris dans un colonialisme "double » : interne, contrôlés par une élite palestinienne impuissante à l'initiative politique; et externe, victimes d'une colonisation israélienne totale.

\section{Conclusion}

La distinction entre « fonction enseignante » et « condition enseignante » offre un outillage conceptuel sensible aux enjeux qui façonnent le travail enseignant dans le contexte palestinien. Elle permet d'interroger le réductionnisme qui afflige les discours courants sur la fonction enseignante, comme les décrit Shinn (2012). Ces discours négligent les complexités qui sous-tendent toute récupération du travail enseignant pour une société palestinienne ravagée et subjuguée par plus d'un siècle de colonialisme.

Comme fonction, le travail enseignant palestinien a émergé à la fois à la suite d'initiatives privées et par le pouvoir en place. Depuis la Nakba, son essor quantitatif eut lieu sous condition apatride et sous des régimes coloniaux. Ces derniers contrarient l'autodétermination palestinienne par le biais de systèmes de gouvernance perçus comme exogènes et fondés sur un patronage politique et social. Ironiquement, 
l'essor le plus marqué de la fonction eut lieu dans les "écoles du gouvernement ", alors que l'absence d'un État (en Cisjordanie et à Gaza) et la subordination politico-culturelle (en Israël) perdurent. Je maintiens que cette donne institutionnalise une tension relationnelle d'aliénation, accentuant la méfiance et la résistance - explicites ou implicites, selon les contextes - entre la fonction enseignante et le pouvoir en place.

Comme condition, les réalités vécues du travail enseignant palestinien ont connu des changements dans ses clivages sociaux. La dominance des élites sociales masculines s'est éclipsée durant la colonisation britannique avec l'entrée des femmes, et surtout des classes sociales moyennes, rurales et défavorisées, pour qui l'enseignement représentait une première base de mobilité dans une société colonisée, en pleine urbanisation, connaissant l'émergence d'un mouvement national. À la suite de la Nakba, la condition apatride et l'expérience exilique transformèrent le travail enseignant en activité transrégionale. L'entrée d'enseignants et d'enseignantes d'origine réfugiée et de classes sociales défavorisées représentait leur première position à la fois dans le champ économique et dans un champ politique reconfiguré par rapport à l'avant-Nakba. La féminisation de la fonction enseignante fut accompagnée par une précarisation économique et sociale considérable de la fonction enseignante.

Deux questions interdépendantes ont guidé ma réflexion sur la condition enseignante palestinienne. La première a problématisé les tensions entre insertion politique des enseignants et enseignantes et leurs pratiques dans la salle de classe. Ce fil rouge témoigne de la perméabilité entre le champ éducatif et les flux et reflux des réseaux qui jonchent le champ politique. Il témoigne aussi de la vulnérabilité institutionnelle de l'école et de la salle de classe, suggérant que « la coupure entre le monde et l'école » (Galvão, 2015) n'a pas été institutionnalisée dans le contexte palestinien. Plutôt, «le brouillage des frontières » entre école et Cité - symboliquement, cet ordre politique humain et les luttes qui l'attisent - est constant. Différentes parties cherchent à imposer la clôture de l'école, ou à assurer son ouverture, pour favoriser ou perturber les agencements entre les champs politique et éducatif. La Cité « déborde » dans l'école palestinienne, reflétant, d'une part,l'acharnement des pouvoirs en place à subjuguer le travail enseignant à des projets coloniaux (internes et externes); et, d'autre part, la réticence des enseignants et enseignantes à être réduits à ce que j'appelle un "pédagogisme désincarné », sans sens ni vision. Veronese et al. (2018) indiquent que les enseignants et enseignantes dans la société palestinienne en Israël et dans les Territoires palestiniens occupés «se sentent exclus des processus de prise de décisions tant à l'intérieur qu'à l'extérieur des écoles, et leur développement professionnel est limité en raison du racisme et de la répression généralisés et institutionnalisés ", accentuant leur "sentiment de marginalisation » (p. 24).

La deuxième question a touché aux lectures à contrepoint que font les enseignants et enseignantes des violences vécues. Ces lectures montrent la réinscription du sujet dans son autobiographie, comme cheminement professionnel incarné. En outre, elles illustrent «comment la culture enseignante s'immisce dans les projets politiques nationaux » et les liens qu'elles établissent entre école, enseignement et Cité (Col, 2008). Ceci étant, les lectures à contrepoint effectuées durant la colonisation britannique, et subséquemment en exil, par exemple par Khalaf, Khaled et Jabra dans les années 1950-1960 ou encore durant l'Intifada captent cette capacité des enseignants et enseignantes de "pouvoir-commencer " (Arendt, 1995), de faire face à l'adversité et de se diriger vers de nouveaux horizons de possibilité. En contraste, depuis les années 1990, les lectures des enseignants et enseignantes en Israël et dans les 
Territoires palestiniens occupés suggèrent un rétrécissement considérable de leur capacité d'aller audelà du chiasme qui s’immisce entre école et Cité avec la présence régulatrice croissante des pouvoirs en place, comme si les enseignants et enseignantes étaient dorénavant condamnés à vivre comme des étrangers, à la lumière de normes « professionnelles » sisyphiennes situées en dehors de leur temps et de leurs espaces.

\section{NOTES}

1 La composante identitaire commune entre enseignants, enseignantes et élèves perdure parmi les 21571 membres du corps enseignant dans les 692 écoles de l'UNRWA, servant plus d'un demi-million d'élèves dans 58 camps de réfugiés palestiniens à travers le Moyen-Orient (2016). Souad Srej, réfugiée et directrice de l'école primaire de l'UNRWA, « Haïfa » (Beyrouth, Liban), l'exprima ainsi : « nous sommes tous des réfugiés », en se référant à l'accueil d'élèves palestiniens affectés par les violences armées auxquelles leurs camps sont exposés au Liban et, actuellement, en Syrie. Source : UN Volontaires, https://www.unv.org/fr/actualités/au-liban-des-professeurs-volontaires-de-l'onuenseignent-dans-les-écoles-de-lunrwa-aux

2 Un tableau peint en 1989 par Tamam Al-Akhal (née en 1935) - réfugiée, ancienne enseignante dans une école pour filles à Beyrouth, activiste dans le cadre de l'OLP et artiste palestinienne - capte bien par ses symbolismes l'activisme des enseignantes et enseignants durant l'Intifada. Dans son tableau, intitulé « Dans l'ombre de l'Intifada " (huile sur toile, 75 x $60 \mathrm{~cm}$ ), Al-Akhal mit l'accent sur le rôle de la fonction enseignante dans l'inculcation d'un esprit de résistance non violente contre l'occupation militaire israélienne (dans le tableau, la plupart des protagonistes tournent le dos au feu des tirs représenté dans la partie gauche du tableau ou bien ils en sont distanciés). La résistance à l'occupation est imaginée non point par rapport aux pierres - symbole iconique de la confrontation avec l'armée israélienne durant l'Intifada - même si ceux-ci jonchent le tableau entièrement. Plutôt, c'est la capacité des jeunes Palestiniens de reconstruire leur vielle maison commune - malgré son état délabré et ruiné - en s'organisant, en étudiant et en plantant ces oliviers, symbole de paix, de vie, de fortitude et de pérennité de la nation. Dans cet espace artistique, les enseignants - homme et femme - évoquent un rôle de dirigeants par leur passion, portés par un zèle et un engagement solidaire (dans le tableau, remarquez leurs poings fermés, lancés vers l'action; les deux enseignants sont aussi les seuls qui parlent, avec leur bouche clairement ouverte, alors que les étudiants - filles et garçons, assis côte à côte - écoutent intensément). (Voir sur le site de l'artiste à l'adresse http://www.ismail-shammout.com/cms/ tamam-alakhal-gallery.html, le tableau tam-81, dans la section des années 1980-1989.) Ce tableau contraste avec la représentation bien plus récente de l'enseignant sur une paire de timbres postaux, émis par l'AP en 2018. Sur ce timbre, la forme stylisée d'un enseignant (à déduire par la forme) est drapée du drapeau palestinien, en posture de mobilisation nationale, pointant - avec autorité - un bâton vers une carte de la Palestine. (Voir le site à l'adresse http://www.palstamps.eu/PSRamallah.htm, émission numéro 397 intitulée « Palestinian Teachers' Day ».)

\section{Références}

Al-Haq. (1990). A nation under siege: Al-Haq annual report on human rights in the occupied Palestinian territories - 1989. Ramallah : Al-Haq. http://dx.doi.org/10.1163/2210-7975 hrd-0965-0065

Al-Zaroo, S. (1988). L'enseignement sous l'occupation, 1967-1987. Hébron : Association des Universitaires. (Arabe)

Arendt, H. (1995). Qu'est-ce que la politique? Paris : Seuil.

Bateson, M. C. (1989). Composing a life. New York, NY : Grove Press.

Boullata, K. (2009). Palestinian art, 1850-2005. Londres : Al-Saqi.

Col, C. (2008). Les enseignants produisent-ils ou reproduisent-ils du lien? Construction des médiations socio-scolaires à l'école primaire. Pensée plurielle, (18), 57-72. http://dx.doi.org/10.3917/pp.018.0057

Davis, R. (2003). Commemorating education: recollections of the Arab College in Jerusalem, 1918-1948. Comparative Studies in South Asia, Africa and the Middle East, 23(1-2), 190-204. http://dx.doi.org/10.1215/1089201x-23-1-2-190

Fairclough, A. (2007). A class of their own: Black teachers in the segregated South. Cambridge, MA : Harvard University Press.

Galvão, I. (2015). Au-delà des murs : penser les frontières entre l'école et la Cité. Le sujet dans la Cité, (6), 59-70. 
Graham-Brown, S. (1984). Education, repression, liberation: Palestinians. Londres : World University Service.

Hauser, J., Lindner, C. B. et Möller, E. (2016). Entangled education: foreign and local schools in Ottoman Syria and Mandate Lebanon (19th-20th centuries). Beyrouth : Orient-Institut.

Jabra, I. J. (1997). Portrait de l'exilé palestinien en écrivain. Revue d'études palestiniennes, (63), 3-11.

Khalaf, S. [Abou Iyad]. (1978). Palestinien sans patrie. Paris : Fayolle.

Khaled, L. (1973). Mon people vivra. Paris : Gallimard.

Khoury-Watad, K. (2008). Les répercussions du conflit israélo-palestinien sur le rôle de l'enseignant arabe en Israël. $B a-$ mikhlala: mehkar, 'iyoun, ve-yetsira [Au Collège : Recherche, revue, et créativité], 21, 263-284. (Hébreu)

Lanoue, E. (2006). Éducation, violences et conflits en Afrique subsaharienne. Dans M. Pilon (dir.), Défis du développement en Afrique subsabarienne : l'éducation en jeu (p. 223-242). Paris : CEPED.

Masalha, N. (2012). The Palestine Nakba. Londres : Zed Books.

Mazawi, A. E. (2005). Pathologizing the schooling experience of Negev Bedouin Arab children in Israel. Dans C. M. Shields, R. Bishop, et A. E. Mazawi, Pathologizing practices: The impact of deficit thinking in education (p. 85117). New York, NY : Peter Lang.

Mazawi, A. E. (2017). School textbooks and entanglements of the 'colonial present' in Israel and Palestine. Dans C. Borg et M. Grech (dir.), Pedagogy, politics and philosophy of peace (p. 160-180). New York, NY : Bloomsbury. http://dx.doi.org/10.5040/9781474282826.0019

Mills, C. W. (1967). L'imagination sociologique (traduit par P. Clinquart). Paris : Maspero.

Moussa, S. (1972). Quelques remarques concernant l'expérience de l'Union générale des enseignants palestiniens. Shou'oun Filastiniya [Affaires palestiniennes], 16,150-162. (Arabe)

Oolbekkink-Marchand, H. W., Hadar, L. L., Smith, K., Helleve, I. et Ulvik, M. (2017). Teachers' perceived professional space and their agency. Teaching and Teacher Education, 62, 37-46. http://dx.doi.org/10.1016/j.tate.2016.11.005

Palestinian Central Bureau of Statistics, and Ministry of Education and Higher Education (1995). Education statistics yearbook, 1994/1995. Ramallah : West Bank. Repéré à http://www.pcbs.gov.ps/Downloads/book4.pdf

Ricks, T. M. (2008). Khalil Totah: the unknown years. Jerusalem Quarterly, 34, 51-77.

Royaume hachémite de Jordanie. Ministère de l'Éducation. (1966). Rapport annuel sur l'éducation dans les écoles du ministère de l'Éducation durant l'année scolaire 1965-1966. Amman : Ministère de l'Éducation. (Arabe)

Sachs, J. (2016). Teacher professionalism: Why are we still talking about it? Teachers and Teaching, 22(4), 413-425. http:// dx.doi.org/10.1080/13540602.2015.1082732

Sa'di, A. H. (2014). Through surveillance: The genesis of Israeli policies of population management, surveillance and political control towards the Palestinian minority. Manchester : Manchester University Press.

Said, E. W. (1994). Representations of the intellectual. New York, NY : Vintage.

Sayigh, Y. (1997). Armed struggle and the search for state: the Palestinian national movement, 1949-1993. Oxford : Clarendon Press.

Shalhoub-Kevorkian, N. (2010). Palestinians, education, and the Israeli 'industry of fear'. Dans A. E. Mazawi et R. G. Sultana (dir.), World yearbook of education 2010 - Education and the Arab 'world': Political projects, struggles, and geometries of power (p. 335-349). Londres : Routledge. http://dx.doi.org/10.4324/9781315083834-20

Shinn, C. (2012). Teacher education reform in Palestine: Policy challenges amid donor expectations. Comparative Education Review, 56(4), 608-633. http://dx.doi.org/10.1086/667434

State of Israel, Central Bureau of Statistics. (2015). Statistical abstract of Israel. Jérusalem : Central Bureau of Statistics.

State of Palestine, Central Bureau of Statistics. (2016). Palestinians at the end of 2016. Ramallah : Central Bureau of Statistics.

Sultana, R. G. (2006). Education in conflict situations: Palestinian children and distance education in Hebron. Mediterranean Journal of Educational Studies, 11(1), 49-81.

Tardif, M. (2013). La condition enseignante au Québec du XIX au XXI siècle: Une histoire cousue de fils rouges : précarité, injustice et déclin de l'école publique. Laval, QC : Presses de l'Université Laval.

Tardif, M. (2014). Liberté et reconnaissance au cœur de l'enseignement. Relations, (774), 22-23. Repéré à https://www.erudit.org/fr/revues/rel/2014-n774-rel01507/72457acl 
Tawil, S. (1998). Rapport final de la réunion, Genève, 15-16 mai 1997. Dans S. Tawil (dir.), La destruction et la reconstruction de l'éducation dans les sociétés perturbées (p. 7-17). Repéré à https://unesdoc.unesco.org/in/rest/annotationSVC/ DownloadWatermarkedAttachment/attach import b61f862c-c915-4e53-b7c6-1fc836db3170? =149377fre.pdf

Tibawi, A. L. (1956). Arab education in mandatory Palestine: a study of three decades of British administration. Londres : Luzac $\&$ Co.

Tsehaye, R. S. (2015). Les stratégies scolaires face aux enjeux normatifs internationaux. Revue Tiers Monde, (223), $183-204$. http://dx.doi.org/10.3917/rtm.223.0183

UNESCO. (1995). Primary and secondary education in the West Bank and Gaza Strip. Overview of the system and needs for the development of the Ministry of Education. Repéré à https://unesdoc.unesco.org/in/rest/annotationSVC/ DownloadWatermarkedAttachment/attach import 68bf2a5f-e969-4721-896a-19a235a35a64? =103049engb.pdf

UNOCHA [United Nations Office for the Coordination of Humanitarian Affairs - Occupied Palestinian Territory]. (2014). Gaza emergency. Situation report (as of 4 September 2014, 08:00 hrs). Repéré à https://www.ochaopt.org/ content/occupied-palestinian-territory-gaza-emergency-situation-report-4-september-2014-0800-hrs

Ushpiz, Y. (1940). Quand l'éducation sera dans les mains du gouvernement. Héd Habinoukb [Écho de l'Éducation], (9-11), 203-208. (Hébreu)

Veronese, G., Pepe, A., Dagdukee, J. et Yaghi, S. (2018). Teaching in conflict settings: dimensions of subjective wellbeing in Arab teachers living in Israel and Palestine. International Journal of Educational Development, 61, 16-26. http://dx.doi.org/10.1016/j.ijedudev.2017.11.009

Wu, J., Cheung, H. Y. et Chan, R. M. C. (2017). Changing definition of teacher professionalism: Autonomy and accountability. Dans I. H. Amzat et N. P. Valdez (dir.), Teacher empowerment toward professional development practices: perspectives across borders (p. 59-70). Singapour : Springer. http://dx.doi.org/10.1007/978-981-10-4151-8 4

\section{Pour citer cet article}

Mazawi, A. E. (2019). Colonialismes, adversités et statut apatride : La condition enseignante dans le contexte palestinien. Formation et profession. 27(1), 37-52. http://dx.doi.org/10.18162/fp.2018.467 